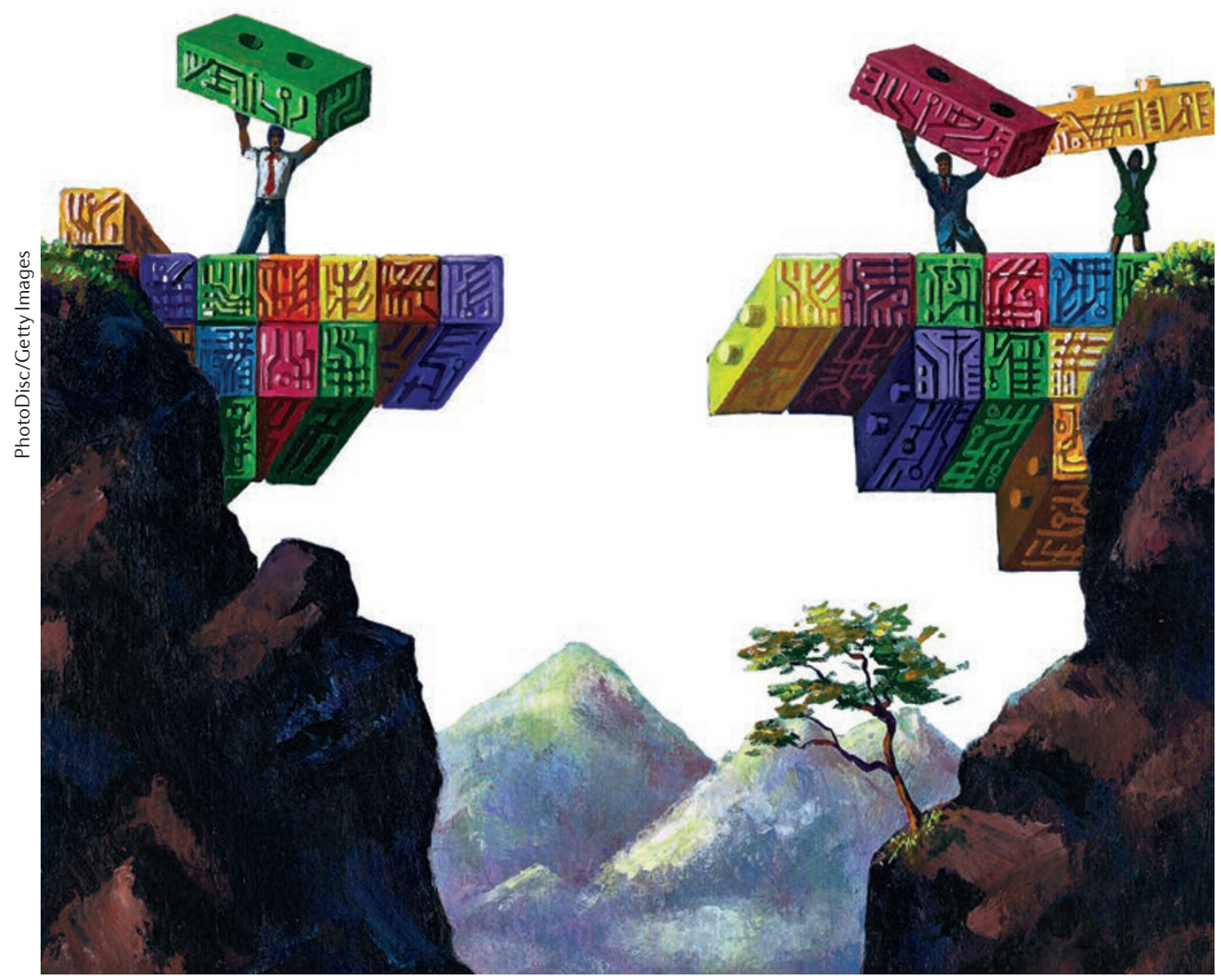

ANTICANCER DRUGS

\section{Brain metastasis bridges the gap}

An estimated $20-40 \%$ of advanced stage cancers metastasize to the brain making brain metastases more common than many primary tumours as well as a key contributor to cancer mortality. A new study in Nature has suggested that targeting gap junction activity could be a successful therapeutic strategy against brain metastases.

Chen et al. began by attempting to explain the surprising observation that cancer cells co-cultured with astrocytes display increased survival when treated with cytotoxic agents, despite the recognized neuralprotective properties of astrocytes. They then identified a heterocellular interaction between astrocytes and brain metastatic cancer cells through the establishment of hexameric connexin hemi-channels known as gap junctions. Immunohistochemistry revealed that the major gap junction protein, connexin 43 (CX43; also known as gap junction protein $\alpha 1$ ) was present at cancer cell-astrocyte interfaces in brain metastases of patients with triple-negative breast cancer and non-small cell lung cancer. Moreover, CX43 was expressed at higher levels compared with primary tumours or normal tissues, indicating a specific increased dependency upon CX43 in brain metastases.

Searching for a cell-cell adhesion molecule that could induce astrocytic gap junction formation, in conjunction with CX43, the authors identified protocadherin 7 (PCDH7), an integral membrane protein found almost exclusively in the brain. PCDH7 was highly expressed in brain metastatic cell lines compared with their parental counterparts and matched bone or lung metastatic derivatives. Consistent with a role for both PCDH7 and CX43 in gap junction assembly, knockdown of either protein resulted in decreased dye transfer from cancer cells to astrocytes in vitro and reduced outgrowth of brain micrometastases in mouse models of breast and lung cancers.

Further experiments revealed the mechanistic details by which cancer cell-astrocyte gap junctions support brain metastases. Firstly, cytosolic double-stranded DNA, which is known to activate the cyclic GMPAMP synthase (cGAS)-stimulator of interferon genes (STING) innate immune response, was detected in cancer cell lines but not in astrocytes. This correlated with a cancer cellspecific increase in 2'3'-cyclic GMPAMP (cGAMP), a second messenger generated from the cGAS-STING pathway. Secondly, increased expression of interferon al (IFNA1) and tumour necrosis factor (TNF) mRNA was detected in astrocytes co-cultured with cancer cells, while increased phosphorylation of signal transducer and activator of transcription 1 (STAT1) and the nuclear factor- $\kappa \mathrm{B}$ (NF-kB) p65 subunit (also known as RELA) was observed in brain metastatic cancer cells co-cultured with astrocytes. The authors concluded that brain metastatic cancer cells use CX43-PCDH7 gap junctions to transfer cGAMP to astrocytes, which in turn activates the STING pathway and generates paracrine signals IFNa and TNF to trigger STAT1 and NF- $\kappa B$ survival pathways in brain metastatic cancer cells.

Importantly, the authors showed that treatment with gap junction inhibitors was sufficient to specifically decrease brain but not lung metastases in xenograft and syngeneic mouse models of metastasis. Furthermore, inducible knockdown of either CX43 or PCDH7 in established brain metastases improved sensitivity to chemotherapy.

Early studies of gap junctions proposed that they were associated with tumour suppression; however, these data highlight that cancer cell dependency on gap junctions is likely to be both context and stage specific.

Anna Dart, Locum Associate Editor, Nature Reviews Cancer

This article is modified from the original in Nat. Rev. Cancer (http://dx.doi.org/10.1038/nrc.2016.64) 